\title{
Primeiros socorros na Educação Física: uma revisão bibliométrica
}

\author{
First aid in Physical Education: an bibliometric review \\ Primeros auxilios en Educación Física: una revisión bibliométrica
}

Recebido: 16/01/2021 | Revisado: 24/01/2021 | Aceito: 27/01/2021 | Publicado: 02/002/2021

\author{
André Luiz Dantas Bezerra \\ ORCID: https://orcid.org/0000-0002-0547-5772 \\ Centro Universitário de Patos, Brasil \\ Faculdade São Francisco da Paraíba, Brasil \\ E-mail: milenanunes@ fiponline.edu.br \\ Larissa de Araújo Batista Suárez \\ ORCID: https://orcid.org/0000-0002-6658-5019 \\ Universidade Católica de Pernambuco, Brasil \\ Faculdade São Francisco da Paraíba, Brasil \\ E-mail: labsuarez@gmail.com \\ Milena Nunes Alves de Sousa \\ ORCID: https://orcid.org/0000-0001-8327-9147 \\ Centro Universitário de Patos, Brasil \\ Faculdade São Francisco da Paraíba, Brasil \\ E-mail: milenanunes@fiponline.edu.br
}

\begin{abstract}
Resumo
Objetivou-se analisar o perfil das publicações sobre primeiros socorros na educação física. Adotou-se o método bibliométrico. A busca foi realizada entre julho de 2020 e janeiro de 2021 na Biblioteca Virtual em Saúde (BVS) a partir dos Descritores em Ciências da Saúde (DeCS) em português: "Primeiros Socorros" e "Educação Física". Identificaram-se 25 publicações, selecionando-se 11 que se adequaram aos critérios de elegibilidade. Os artigos foram publicados entre 2009-2020, majoritariamente na Medical Literature Analysis and Retrieval System Online (63,63\%; $\mathrm{n}=7)$, no idioma inglês $(63,63 \% ; \mathrm{n}=7)$ e em periódicos internacionais $(63,63 \% ; \mathrm{n}=7)$, destacando-se o European Journal of Paediatric Dentistry e a Dental Traumatology, com 18,18\% ( $\mathrm{n}=2)$, cada. Também, a maior parte era da área de odontologia $(72,73 \%$; $n=8)$ e em relação ao Qualis Capes, as revistas transitaram entre A1 e B4. Quanto à autoria, foram 41 autores distintos e apenas um autor polonês destacou-se com duas produções $(18,18 \%$; $n=2)$. Os termos mais citados nos títulos e descritores foram conhecimento $(25,0 \% ; n=12)$, educação $(22,92 \% ; n=11)$ e educação física $(16,47 \% ; \mathrm{n}=8)$ e as temáticas trabalhadas versaram, principalmente, sobre as estratégias de intervenção $(100,0 \% ; \mathrm{n}=11)$ e sobre traumas dentários $(72,73 \% ; \mathrm{n}=8)$. Os achados indicaram que há pouco conhecimento na área, o que demanda a adoção de estratégias educativas para qualificar os profissionais e os estudantes da área de educação física no atendimento de primeiros socorros.
\end{abstract}

Palavras-chave: Primeiros socorros; Educação física e treinamento; Educação em saúde.

\begin{abstract}
The objective of this study was to analyze the profile of publications on first aid in physical education. The bibliometric method was adopted. The search was conducted between July 2020 and January 2021 at the Virtual Health Library from the Descriptors in Health Sciences in Portuguese: "First Aid" and "Physical Education". Twentyfive publications were identified, selecting 11 of which met the eligibility criteria. The articles were published between 2009-2020, mostly in the Medical Literature Analysis and Retrieval System Online (63.63\%; $\mathrm{n}=7$ ), in English $(63.63 \% ; \mathrm{n}=7)$ and in international journals $(63.63 \%$; $\mathrm{n}=7)$, especially the European Journal of Paediatric Dentistry and Dental Traumatology, with $18.18 \%(\mathrm{n}=2)$, each. Also, most were from the area of dentistry $(72.73 \%$; $\mathrm{n}=8$ ) and in relation to Qualis Capes, the journals moved between A1 and B4. As for authorship, there were 41 different authors and only one Polish author stood out with two productions $(18.18 \%$; $n=2)$. The most cited terms in the titles and descriptors were knowledge $(25.0 \% ; \mathrm{n}=12)$, education $(22.92 \% ; \mathrm{n}=11)$ and physical education $(16.47 \%$; $\mathrm{n}=8)$ and the themes worked mainly focused on intervention strategies $(100.0 \% ; \mathrm{n}=11)$ and about dental traumas $(72.73 \% ; n=8)$. The findings indicated that there is little knowledge in the area, which requires the need to adoption of educational strategies to qualify professionals and students in the area of physical education in the care of first aid.
\end{abstract}

Keywords: First aid; Physical education and training; Health education.

\section{Resumen}

El objetivo de este estudio fue analizar el perfil de las publicaciones sobre primeros auxilios en educación física. Se adoptó el método bibliométrico. La búsqueda se llevó a cabo entre julio de 2020 y enero de 2021 en la Biblioteca Virtual de Salud de los Descriptores en Ciencias de la Salud en Portugués: "Primeros Auxilios" y "Educación Física". 
Se identificaron veinticinco publicaciones, seleccionando 11 de las cuales cumplían los criterios de elegibilidad. Los artículos fueron publicados entre 2009-2020, principalmente en el Sistema de Análisis y Recuperación de Literatura Médica en Línea $(63,63 \%$; n-7), en Inglés $(63,63 \% ; n-7)$ y en revistas internacionales $(63,63 \%$; n-7), especialmente el Diario Europeo de Odontología Pediátrica y Traumatología Dental, con 18,18\% (n-2), cada uno. Además, la mayoría eran del área de la odontología (72,73\%; n-8) y en relación con Qualis Capes, los diarios se movieron entre A1 y B4. En cuanto a la autoría, había 41 autores diferentes y sólo un autor polaco se destacó con dos producciones $(18,18 \%$; n2). Los términos más citados en los títulos y descriptores fueron el conocimiento (25,0\%; No 12$)$, educación (22,92\%; n-11) y educación física $(16,47 \% ; n-8)$ y los temas se centraron principalmente en las estrategias de intervención $(100,0 \% ; n-11)$ y sobre traumas dentales $(72,73 \%$; n-8). Los hallazgos indicaron que hay poco conocimiento en la zona, lo que requiere la necesidad de adoptar estrategias educativas para calificar a profesionales y estudiantes en el área de la educación física en el cuidado de primeros auxilios.

Palabras clave: Primeros auxilios; Educación y entrenamiento físico; Educación en salud.

\section{Introdução}

É comum a ocorrência de acidentes, visto que é um evento inesperado. "Os indivíduos em qualquer estágio da vida e nos mais diversos ambientes estão sujeitos a se acidentarem ou sofrerem um mal súbito” (Becker, Molina \& Nunes, 2017, p. 1). Destarte, é possível acidentar-se em um shopping, na rua, em casa e na escola (Souza \& Tibeau, 2008).

No ambiente escolar, os acidentes são comuns e tornam-se uma preocupação no cotidiano das instituições educacionais (Becker, Molina \& Nunes, 2017). Na prática das atividades físicas, quer sejam executadas “em academias, nos parques e, principalmente, na escola, o risco de acidentes aumenta ainda mais” (Souza \& Tibeau, 2008, p. 1).

"Nestas situações, as consequências são proporcionais à gravidade do ocorrido e à qualidade dos primeiros socorros ofertados” (Becker, Molina \& Nunes, 2017, p. 1). Assim sendo, o conhecimento sobre primeiros socorros é fundamental (Coelho, 2015) e compreendem, conforme a Fundação Oswaldo Cruz (Brasil, 2003, p. 8), como...

[...] os cuidados imediatos que devem ser prestados rapidamente a uma pessoa, vítima de acidentes ou de mal súbito, cujo estado físico põe em perigo a sua vida, com o fim de manter as funções vitais e evitar o agravamento de suas condições, aplicando medidas e procedimentos até a chegada de assistência qualificada.

A relevância sobre os primeiros socorros é ainda mais significativa entre os professores de educação física, pois "a busca constante pelo movimento proporcionado pelas aulas de educação física e as dificuldades na supervisão durante outras atividades escolares costuma acarretar em acidentes com lesões variadas" (Cunha, 2010, p. 6).

Nas escolas, quando da ocorrência de acidentes, o professor de educação física geralmente é requerido para aparecer no instante em que acontece uma situação ou acidente com os estudantes (Souza \& Tibeau, 2008). Assim, é primordial que tenha conhecimento e preparo para atuar adequadamente ante aos traumas e lesões (Becker, Molina \& Nunes, 2017).

No mais, nota-se o despreparo de docentes quanto à temática e suporte em primeiros socorros nas escolas (Souza \& Tibeau, 2008; Silva, Costa, Furtado, Tavares \& Costa, 2017). “Os professores não sabem como agir em situações que comprometam a saúde da criança, gerando risco para o estado vital do escolar” (Silva et al., 2017, p. 26).

Corrobora com a assertiva anterior, o estudo de Cunha (2010), o qual objetivando levantar os tipos, características e fatores que provocam situações emergenciais e acidentes em instituição básica de ensino de Florianópolis, constatou que os entrevistados (professores de educação física e servidores) carecem de capacitação em primeiros socorros imediata para desenvolvimento de competências (saberes, habilidades e atitudes).

“A falta de preparo da comunidade escolar e dos professores impede o auxílio na hora em que ocorre o acidente e podem causar consequências graves para o aluno, prejuízos para o professor e para a escola” (Souza \& Tibeau, 2008, p. 1). Portanto, propõe-se analisar o perfil das publicações sobre primeiros socorros na educação física.

O estudo apresenta importância, pois pode indicar caminhos para atuação e efetivação de protocolos para atuação do professor de educação física, bem como evidenciar as temáticas que necessitam ser mais bem exploradas. 


\section{Metodologia}

Estudo bibliométrico, aplicado "por diversos pesquisadores com o intuito de obter um melhor entendimento sobre a literatura de um determinado tema" (Eiras, Tomomitsu, Linares \& Carvalho, 2017, p. 213). Exige que sejam adotadas leis: Bradford (reputação do periódico), Zipf (frequência de palavras-chave) e Lotka (produção por autor) (Chueke \& Amatucci, 2015).

\subsection{Caracterização da amostra}

Para a análise do o perfil das publicações sobre primeiros socorros na educação física, optou-se pela seleção do material na Biblioteca Virtual em Saúde (BVS) do Brasil, disponível no domínio <<http://brasil.bvs.br/>>. Esta base de dados é referência para estudantes e pesquisadores brasileiros, razão para a sua elegibilidade.

A busca, realizada entre julho a dezembro de 2020, foi efetivada a partir dos Descritores em Ciências da Saúde (DeCS) em português: "Primeiros Socorros" e "Educação Física e Treinamento", associados a partir da utilização do operador booleano AND. A triagem dos DeCS aconteceu na homepage <http://decs.bvs.br/>.

Identificaram-se 25 documentos. Como critérios de seletividade, incluíram-se o tipo de documentos, disponibilidade online, artigos independentemente do método e sem recorte temporal, desprezaram-se os trabalhos de conclusão de cursos (monografias, dissertações e teses), os editoriais, manuais e notas técnicas.

\subsection{Análise dos dados}

Os 11 artigos constituintes da amostra foram analisados aos pares em uma fase preliminar e extraídas as informações: autor, ano, título, base de dados, periódico, Qualis Capes, idioma, país e formação do primeiro autor. Os dados extraídos foram submetidos à estatística descritiva simples (frequência absoluta e relativa).

Esta tarefa permitiu identificar o período relacionado ao ano com maior número de publicação, verificar a distribuição dos artigos conforme autores, na prerrogativa de instituir uma elite científica, bem como o periódico em maior evidência. Ademais, foi possível averiguar a análise temática a partir palavras-chave (descritores) disponíveis no resumo e no título dos artigos e constatar as abordagens de maior enfoque nas produções.

Para tanto, a nuvem de palavras foi construída no Wordcloud, um gerador gratuito e disponível virtualmente na homepage https://www.wordclouds.com/. Posteriormente, os dados coletados foram apresentados e sintetizados.

\section{Resultados e Discussão}

De acordo com o material selecionado (Quadro 1), foi possível verificar que os artigos foram publicados entre 20092020, portanto, nos últimos 12 anos. Os anos com maior representatividade foram: 2009, 2013 e 2016, com 18,18\% (n=2), cada. Constatou-se que a base de dados em destaque foi a Medical Literature Analysis and Retrieval System Online (MEDLINE) $(63,63 \% ; n=7)$ e a maioria estava disponível no idioma inglês $(63,63 \%$; n=7). Para Sousa (2020, p. 65), a língua inglesa é a linguagem da ciência/científica e "a MEDLINE é o principal componente do PubMed®, mas também pode ser acessada pela Biblioteca Virtual em Saúde (BVS) do Brasil”.

Reforça-se que a evolução histórica quanto ao número de publicações e os periódicos que abordaram sobre a temática são mensurações estabelecidas pela Lei de Bradford (Chueke \& Amatucci, 2015; Rodrigues \& Viera, 2016). "Enfatiza-se que ao selecionar o material é fundamental priorizar pela qualidade da fonte, pela atualidade [...], idioma (opte por aqueles na língua inglesa, mas não deixe de mostrar resultados de sua região geográfica)" (Sousa, 2020, p. 54).

Quanto à formação dos autores, a maioria era da área de odontologia $(72,73 \%$; n=8) e eram pesquisadores brasileiros $(45,45 \% ; n=5)$. Uma crítica que se faz é o fato de que poucas pesquisas eram, especificadamente, de autores da educação 
física. Estudo com o objetivo de "analisar a produção do conhecimento sobre o cotidiano da Educação Física (EF) escolar" verificou que os temas correntes abordados eram "diagnóstico didático e formação profissional" e os menos contemplados "violência na escola e políticas públicas". Os autores concluíram que "os aspectos constitutivos do cotidiano escolar merecem maior aprofundamento devido à sua complexidade" (Maldonado, Silva \& Miranda, 2014, p. 1373).

Entretanto, foi possível constatar que a maior parte das publicações estava em periódicos internacionais (63,63\%; $\mathrm{n}=7$ ), destacando-se o European Journal of Paediatric Dentistry e a Dental Traumatology, com 18,18\% (n=2), cada. Em relação ao Qualis Capes, as revistas transitaram entre A1 e B4. As de menor qualificação foram as nacionais (B3 e B4). O Qualis “é um sistema 'métrico' brasileiro utilizado para avaliar os periódicos” (Sousa, Almeida, Melo \& Almeida, 2019, p. 90).

O novo estrato, proposto em 2019, classifica as publicações entre A1-A4, B1-B4 e C. Quanto mais próximo do A, mas bem avaliado. Esta qualificação é útil para ponderar sobre a credibilidade do material que está sendo publicado pelos periódicos (Sousa, 2020). Importante frisar que a maioria do material selecionado foi publicada em periódico A (A1, A3 e A4) $(45,54 \% ; n=6)$.

Quanto à autoria, foram 41 autores distintos e apenas o autor Emerich, da Polônia, destacou-se com 18,18\% (n=2) das produções. Os autores mais produtivos devem ser mensurados como determina a Lei de Lotka (Chueke \& Amatucci, 2015; Rodrigues \& Viera, 2016).

No mais, a realidade apresentada mostrou que não há uma elite científica relacionada ao objeto de estudo, entretanto, evidencia a rede de colaboração científica entre os pesquisadores. "As redes de colaboração técnico-científica são formadas a partir de pelos menos dois atores" (Sousa, 2020, p. 33).

Para a autora citada anteriormente, é crescente a colaboração entre os pesquisadores e esta conexão traz benefícios diversos, tais como: interatividade, economia de tempo, aumento na produção acadêmica e científica, entre outros. Destarte, na acepção de Sousa et al. (2019, p. 90), "é fundamental tecer redes de relacionamentos entre os pesquisadores". 
Quadro 1. Caracterização geral dos estudos selecionados quanto ao autor, ano, título, base de dados, periódico, Qualis Capes, idioma, formação do primeiro autor e país.

\begin{tabular}{|c|c|c|c|c|c|}
\hline $\begin{array}{c}\text { Autores e } \\
\text { Ano }\end{array}$ & Título & $\begin{array}{c}\text { Base de } \\
\text { Dados }\end{array}$ & $\begin{array}{c}\text { Periódico e } \\
\text { Qualis Capes }\end{array}$ & Idioma & $\begin{array}{c}\text { Formação do } \\
\text { primeiro autor } \\
\text { e país }\end{array}$ \\
\hline $\begin{array}{l}\text { Oleszki e } \\
\text { Emerich } \\
(2015)\end{array}$ & $\begin{array}{l}\text { How to proceed in case of tooth } \\
\text { avulsion: state of student } \\
\text { knowledge }\end{array}$ & MEDLINE & $\begin{array}{l}\text { European Journal } \\
\text { of Paediatric } \\
\text { Dentistry } \\
\text { A4 }\end{array}$ & Inglês & $\begin{array}{l}\text { Odontologia } \\
\text { Polônia }\end{array}$ \\
\hline $\begin{array}{c}\text { Emerich, } \\
\text { Wlodarczyk e } \\
\text { Ziolkowski } \\
\text { (2013) }\end{array}$ & $\begin{array}{l}\text { Education of Sport University } \\
\text { students regarding first-aid } \\
\text { procedures after dental trauma }\end{array}$ & MEDLINE & $\begin{array}{l}\text { European Journal } \\
\text { of Paediatric } \\
\text { Dentistry } \\
\text { A4 }\end{array}$ & Inglês & $\begin{array}{l}\text { Odontologia } \\
\text { Polônia }\end{array}$ \\
\hline $\begin{array}{l}\text { Donaldson e } \\
\text { Finch (2012) }\end{array}$ & $\begin{array}{c}\text { Identifying context-specific } \\
\text { competencies required by } \\
\text { community Australian Football } \\
\text { sports trainers }\end{array}$ & MEDLINE & $\begin{array}{c}\text { British Journal of } \\
\text { Sports Medicine } \\
\text { Al }\end{array}$ & Inglês & $\begin{array}{c}\text { Educação Física } \\
\text { Austrália }\end{array}$ \\
\hline $\begin{array}{c}\text { Vergotine e } \\
\text { Govoni (2010) }\end{array}$ & $\begin{array}{c}\text { Public school educator's } \\
\text { knowledge of initial management } \\
\text { of dental trauma }\end{array}$ & MEDLINE & $\begin{array}{c}\text { Dental } \\
\text { Traumatology } \\
\text { A3 }\end{array}$ & Inglês & $\begin{array}{c}\text { Odontologia } \\
\text { Estados Unidos }\end{array}$ \\
\hline $\begin{array}{l}\text { Antunes, } \\
\text { Souza, } \\
\text { Gonçalves, } \\
\text { Crespo e } \\
\text { Antunes } \\
(2016)\end{array}$ & $\begin{array}{c}\text { Trauma dental e protetor bucal: } \\
\text { conhecimento e atitudes em } \\
\text { estudantes de graduação em } \\
\text { Educação Física }\end{array}$ & LILACS & $\begin{array}{c}\text { Revista Brasileira } \\
\text { de Educação Física } \\
\text { e Esporte } \\
\text { B3 }\end{array}$ & Português & $\begin{array}{l}\text { Odontologia } \\
\text { Brasil }\end{array}$ \\
\hline $\begin{array}{l}\text { Francisco, } \\
\text { Amaral, } \\
\text { Vieira, Pereira } \\
\text { e Braga } \\
\text { (2016) } \\
\end{array}$ & $\begin{array}{l}\text { Conhecimento de estudantes de } \\
\text { Educação Física de Juazeiro do } \\
\text { Norte-CE sobre o atendimento } \\
\text { emergencial ao trauma dental, } \\
\text { avulsão e reimplante dental }\end{array}$ & LILACS & $\begin{array}{l}\text { Journal of the } \\
\text { Health Sciences } \\
\text { Institute } \\
\text { B4 }\end{array}$ & Português & $\begin{array}{c}\text { Odontologia } \\
\text { Brasil }\end{array}$ \\
\hline $\begin{array}{c}\text { Jorge et al. } \\
\text { (2009) }\end{array}$ & $\begin{array}{l}\text { Knowledge of teachers and } \\
\text { students in physical education's } \\
\text { faculties regarding first-aid } \\
\text { measures for tooth avulsion and } \\
\text { replantation }\end{array}$ & MEDLINE & $\begin{array}{c}\text { Dental } \\
\text { Traumatology } \\
\text { A3 }\end{array}$ & Inglês & $\begin{array}{c}\text { Odontologia } \\
\text { Brasil }\end{array}$ \\
\hline $\begin{array}{l}\text { Mohandas e } \\
\text { Chandan } \\
\text { (2009) }\end{array}$ & $\begin{array}{c}\text { Knowledge, attitude and } \\
\text { practice in emergency } \\
\text { management of dental injury } \\
\text { among physical education } \\
\text { teachers: a survey in Bangalore } \\
\text { urban schools }\end{array}$ & MEDLINE & $\begin{array}{l}\text { Journal of Indian } \\
\text { Society of } \\
\text { Pedodontics and } \\
\text { Preventive } \\
\text { Dentistry } \\
\text { B1 } \\
\end{array}$ & Inglês & $\begin{array}{l}\text { Odontologia } \\
\text { India }\end{array}$ \\
\hline $\begin{array}{c}\text { Lempke, } \\
\text { Schmidt e } \\
\text { Lynall (2020) }\end{array}$ & $\begin{array}{c}\text { Athletic Trainers' Concussion- } \\
\text { Assessment and Concussion- } \\
\text { Management Practices: An } \\
\text { Update }\end{array}$ & MEDLINE & $\begin{array}{l}\text { Journal of Athletic } \\
\text { Training } \\
\text { A1 }\end{array}$ & Inglês & $\begin{array}{l}\text { Educação Física } \\
\text { Estados Unidos }\end{array}$ \\
\hline $\begin{array}{l}\text { Silva et al. } \\
\text { (2013) }\end{array}$ & $\begin{array}{c}\text { Conhecimento de alunos do } \\
\text { curso de Educação Física sobre } \\
\text { avulsão dentária }\end{array}$ & LILACS & $\begin{array}{c}\text { Arquivos em } \\
\text { Odontologia } \\
\text { B4 } \\
\end{array}$ & Português & $\begin{array}{c}\text { Odontologia } \\
\text { Brasil }\end{array}$ \\
\hline $\begin{array}{l}\text { Santos et al. } \\
\text { (2011) }\end{array}$ & $\begin{array}{l}\text { A paródia: uma estratégia } \\
\text { educativa para conhecimentos } \\
\text { relacionados à saúde }\end{array}$ & LILACS & $\begin{array}{l}\text { Revista Brasileira } \\
\text { de Ciência e } \\
\text { Movimento } \\
\text { B3 }\end{array}$ & Português & $\begin{array}{c}\text { Educação Física } \\
\text { Brasil }\end{array}$ \\
\hline
\end{tabular}

Fonte: Dados de Pesquisa (2020). 
Referindo-se a caracterização geral dos estudos selecionados quanto à metodologia e o trauma que demanda primeiros socorros em atividades físicas/esportivas, constatou-se que quase a totalidade realizou estudos transversais $(90,91 \%$; $=10)$ e o trauma mais abordado foram os dentários $(72,73 \%$; $=8)$, em que a avulsão foi o mais citado.

Os estudos transversais são estudos observacionais e os mais usados na área de epidemiologia, de baixo custo e execução fácil, contudo, são estudos de prevalência, não sendo possível estimar a razão do problema (Montenegro, Sousa, Medeiros, Santos \& Sousa, 2016).

Quanto aos traumatismos, os mesmos podem variar entre leves (fratura do esmalte) até mais severos (fratura óssea) (Losso, Tavares, Bertoli \& Baratto-Filho, 2011). Os autores ainda expõem que entre os inúmeros traumas odontológicos, a avulsão dental é o deslocamento do dente na sua cavidade e o mais comum nas escolas durante as atividades esportivas e recreativas.

A prevalência encontrada é alta, aproximadamente 22,5\% e maior no sexo masculino (Traebert, Marcon \& Lacerda, 2010). Análise realizada em serviço de urgência de João Pessoa-PB identificou que, entre 501 prontuários selecionados a partir de amostra não-probabilística, 103 (20,56\%) eram de ocorrências clínicas odontológicas (Pereira, Oliveira, Farias, \& Araújo, 2020).

Afeta mais crianças e adolescentes, uma fonte de "problemas sociais, psicológicos, comportamentais, funcionais e estéticos" (Moreira, Figueiredo, Colodetti \& Miranda, 2015, p. 56). Os autores ainda citam que é uma emergência, um problema sério, o qual necessita de atendimento imediato e especializado.

Quadro 2. Caracterização geral dos estudos selecionados quanto à metodologia e o trauma que demanda primeiros socorros em atividades físicas/esportivas.

\begin{tabular}{|c|c|c|}
\hline Autores e Ano & Método & Trauma \\
\hline Oleszki e Emerich (2015) & Transversal & Lesão dentária \\
\hline Emerich, Wlodarczyk e Ziolkowski (2013) & Transversal & Lesões em geral \\
\hline Donaldson e Finch (2012) & Transversal & Trauma dentário \\
\hline Vergotine e Govoni (2010) & Transversal & Trauma dentário \\
\hline Antunes et al. (2016) & Transversal & Avulsão dentária \\
\hline Francisco et al. (2016) & Transversal & Lesão dentária \\
\hline Jorge et al. (2009) & Transversal & Concussão cerebral \\
\hline Mohandas e Chandan (2009) & Transversal & Avulsão dentária \\
\hline Lempke, Schmidt e Lynall (2020) & Transversal & Não especificado \\
\hline Silva et al. (2013) & Transversal \\
\hline
\end{tabular}

Fonte: Dados de Pesquisa (2020).

Ao considerar os 48 termos citados nos títulos e descritores (Figura 1), os quais apresentaram 112 aparições, os vocábulos que se destacaram na nuvem de palavras foram: conhecimento $(n=12)$, educação $(n=11)$, educação física ( $n=8)$, avulsão dentária, trauma $(n=6)$, aluno $(n=6)$, gestão $(n=4)$ e saúde $(n=4)$. O uso de ferramentas para mineração textual, como as disponíveis para a elaboração de nuvens de palavras, pode corroborar com resultados mais ágeis, inteligentes e precisos (Freitas, Neves \& Gonçalves, 2018).

Para os autores, na nuvem, cada termo/palavra tem sua dimensão e veemência de cor conforme a significância do corpus textual (Freitas, Neves \& Gonçalves, 2018, p. 426). Importante salientar que, embora esta bibliometria faça referência ao objeto de estudo "primeiros socorros", na nuvem, o vocábulo apareceu apenas três vezes, daí o tamanho estar menor que os temos anteriormente citados. Apesar do exposto, o dado é uma fonte importante, pois indica para a concepção de bens e outras inovações (Rodrigues \& Viera, 2016). 
Figura 1. Nuvem de palavras.

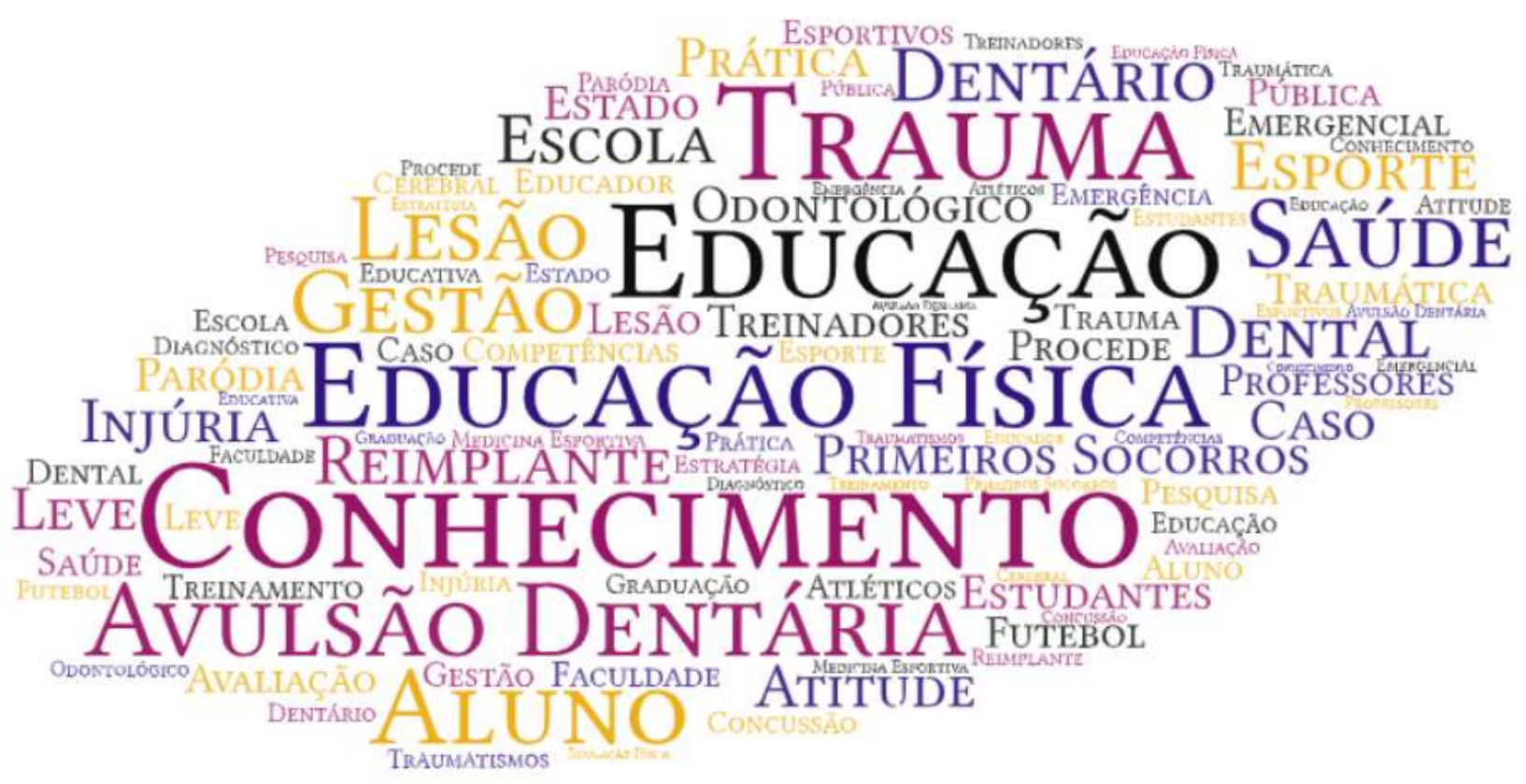

Fonte: Dados de Pesquisa (2020).

Quanto às abordagens de maior enfoque nas produções sobre primeiros socorros na educação física, as categorias contempladas foram: competências $(18,18 \% ; n=2)$, conhecimentos $(72,73 \% ; n=8)$ e estratégias de intervenção $(100 \% ; n=11)$. Esta última abordagem fora citada por todos os artigos selecionados. Estes resultados atendem a outra lei da bibliometria, a Lei de Zipf (Chueke \& Amatucci, 2015; Rodrigues \& Viera, 2016). 
Quadro 3. Categorização e Subcategorização dos estudos: perfil das publicações sobre primeiros socorros na educação física.

\begin{tabular}{|c|c|c|c|c|}
\hline Categorias & Subcategorias & Autores e Ano & n & $\%$ \\
\hline $\begin{array}{l}\text { Competências } \\
(18,18 \% ; n=2)\end{array}$ & Competências exigidas & $\begin{array}{c}\text { Donaldson e Finch (2012) } \\
\text { Lempke, Schmidt e Lynall (2020) }\end{array}$ & 2 & 18,18 \\
\hline \multirow[t]{3}{*}{$\begin{array}{l}\text { Conhecimentos } \\
(72,73 \% ; n=8)\end{array}$} & Baixo & $\begin{array}{c}\text { Antunes et al. (2016) } \\
\text { Emerich, Wlodarczyk e Ziolkowski (2013) } \\
\text { Francisco et al. (2016) } \\
\text { Jorge et al. (2009) } \\
\text { Mohandas e Chandan (2009) } \\
\text { Oleszki e Ewicz (2015) } \\
\text { Silva et al. (2013) } \\
\text { Vergotine e Govoni (2010) }\end{array}$ & 8 & 72,73 \\
\hline & Moderado & - & 0 & 0,0 \\
\hline & Alto & - & 0 & 0,0 \\
\hline \multirow{6}{*}{$\begin{array}{c}\text { Estratégias de } \\
\text { intervenção }(100,0 \% \text {; } \\
n=11)\end{array}$} & Tarefa educacional & Emerich, Wlodarczyk e Ziolkowski (2013) & 1 & 7,69 \\
\hline & $\begin{array}{c}\text { Programas de educação } \\
\text { (palestras, } \\
\text { treinamentos, } \\
\text { campanhas) }\end{array}$ & $\begin{array}{c}\text { Oleszki e Ewicz (2015) } \\
\text { Emerich, Wlodarczyk e Ziolkowski (2013) } \\
\text { Donaldson e Finch (2012) } \\
\text { Vergotine e Govoni (2010) } \\
\text { Antunes et al. (2016) } \\
\text { Francisco et al. (2016) } \\
\text { Mohandas e Chandan (2009) }\end{array}$ & 7 & 53,86 \\
\hline & $\begin{array}{c}\text { Inclusão da temática no } \\
\text { currículo }\end{array}$ & Francisco et al. (2016) & 1 & 7,69 \\
\hline & Uso de Música/paródia & Santos et al. (2011) & 1 & 7,69 \\
\hline & $\begin{array}{c}\text { Avaliações } \\
\text { multidimensionais }\end{array}$ & Lempke, Schmidt e Lynall (2020) & 1 & 7,69 \\
\hline & $\begin{array}{c}\text { Uso de protetores } \\
\text { bucais }\end{array}$ & $\begin{array}{c}\text { Antunes et al. (2016) } \\
\text { Jorge et al. (2009) }\end{array}$ & 2 & 15,38 \\
\hline
\end{tabular}

Nota: cada categoria foi quantificada por número total de artigos $(n=11 ; 100 \%)$ e as subcatergorias pela ocorrência.

Fonte: Dados de Pesquisa (2020).

A partir das abordagens apresentadas, puderam-se tecer ponderações mediante o material selecionado. Na categoria competência, pesquisa propondo alcançar um consenso especializado sobre as competências exigidas pelos treinadores esportivos comunitários do Futebol Australiano, foi possível classificá-las em essencial, esperada, ideal ou não necessária. Portanto, mais da metade endossaram que, entre as competências essenciais ou esperadas destacam-se a gestão de emergência, avaliação de lesões e doenças e manejo imediato, prevenção de lesões e gerenciamento de riscos (Donaldson \& Finch, 2012).

Os autores outrora citados, concluíram que os treinadores esportivos são membros importantes das equipes de primeiros socorros em campo, fornecendo apoio tanto aos jogadores lesionados quanto a outros profissionais de medicina esportiva. No mais, treinadores atléticos precisam melhorar a sua prática, a partir de competências próprias (exame clínico, ferramentas de avaliação padronizadas e outras) (Lempke, Schmidt \& Lynall, 2020).

Por conseguinte, quanto à categoria conhecimentos, estudo propondo comparar o conhecimento de estudantes de esportes, de medicina e demais universitários frequentadores de academias evidenciou baixo conhecimento dos mesmos quanto à avulsão do dente e a necessidade de reimplantação imediata; importância do tempo no prognóstico após a avulsão dentária; e meio de transporte recomendado para um dente avulso (Oleszki \& Emerich, 2015). Este quadro reflete baixo conhecimento sobre as lesões odontológicas comuns em atividades físicas e o atendimento de primeiros socorros.

De modo análogo, outra pesquisa indicou baixo nível de conhecimento inicial dos estudantes de educação física sobre medidas de primeiros socorros no caso de trauma dentário, entretanto, após atividade educativa o saber melhorou significativamente (Emerich, Wlodarczyk \& Ziolkowski, 2013). Ainda, investigação realizada nos Estados Unidos quanto à gestão do trauma bucal com professores de educação física/treinadores e docentes acadêmicos de outras áreas do ensino médio 
constataram que o conhecimento sobre a gestão inicial do trauma dentário foi baixo para ambos os grupos (Vergotine \& Govoni, 2010).

Objetivando "avaliar o conhecimento e as atitudes de estudantes de Educação Física de Universidades públicas do Estado do Rio de Janeiro, Brasil, em relação ao traumatismo dentário e uso de protetor bucal”, Antunes et al. (2016) evidenciaram que apenas 3,21\% dos entrevistados, de 373 universitários, receberam informações sobre trauma dentário e uso do protetor bucal durante o período de formação, apesar da disciplina de primeiros socorros fazer parte da matriz curricular. No mais, a maioria não sabia a conduta frente a uma avulsão dentária, manuseio e transporte do dente.

No nordeste, na cidade de Juazeiro do Norte-CE, constatou-se conhecimento insuficiente entre 120 estudantes de educação física sobre o atendimento emergencial em situação de trauma dentário (Francisco et al., 2016). Em João Pessoa também foi visualizado resultado análogo. A partir da abordagem de 70 acadêmicos de Educação Física, foi percebido que a maioria possuía saber limitado e desconhecia os procedimentos para atuação em casos de avulsão dentária (Silva et al., 2013).

Pesquisa em Belo Horizonte (SP), com estudantes e professores de educação física, no qual dos 442 indivíduos, 53 eram docentes e 389 discentes, verificou que em ambos os grupos há desconhecimento sobre as medidas de primeiros socorros para avulsão de dente, ademais, não usam ou indicam a utilização de protetores bucais durante atividades físicas/esportivas (Jorge et al., 2009).

Em Bangalore, Índia, estudo demonstrou o nível de Conhecimento, Atitude e Prática dos Professores de Educação Física quanto à gestão emergencial de lesões odontológicas. Apesar de 95\%, entre 580 professores de 700 escolas locais possuírem formação em educação física e em primeiros socorros, a pesquisa sinalizou para a falta de conhecimento e prática entre os professores de educação física quanto à gestão emergencial do trauma dentário (Mohandas \& Chandan, 2009).

Por fim, quanto à categoria estratégias de intervenções na área de primeiros socorros, as sugestões estão apresentadas no quadro 4. Entretanto, as atividades educativas foram as mais sugeridas (palestras, treinamentos, campanhas educativas).

Quadro 4. Caracterização das estratégias de intervenções em primeiros socorros.

\begin{tabular}{|c|c|}
\hline Autor /ano & Estratégias de intervenção em primeiros socorros \\
\hline $\begin{array}{l}\text { Oleszki e Emerich } \\
\qquad(2015)\end{array}$ & $\begin{array}{l}\text { Implantação de programas de educação adequados sobre o tema de lesões orofaciais } \\
\text { relacionadas ao esporte para todos os envolvidos (atletas, jogadores e treinadores), objetivando } \\
\text { oferecer ajuda imediata no local de um acidente. }\end{array}$ \\
\hline $\begin{array}{l}\text { Emerich, Wlodarczyk e } \\
\text { Ziolkowski (2013) }\end{array}$ & Realização de palestra de 30 minutos (clara e concisa) e uma tarefa educacional extra. \\
\hline $\begin{array}{l}\text { Donaldson e Finch } \\
\text { (2012) }\end{array}$ & $\begin{array}{l}\text { Com base nas competências identificadas, devem-se estruturar programas de educação de } \\
\text { treinadores esportivos, com temas voltados aos primeiros socorros. }\end{array}$ \\
\hline $\begin{array}{l}\text { Vergotine e Govoni } \\
(2010)\end{array}$ & $\begin{array}{l}\text { Campanhas educativas sobre traumatismo dentário devem ser direcionadas a todos os } \\
\text { professores. }\end{array}$ \\
\hline Antunes et al. (2016) & $\begin{array}{l}\text { Sugere-se que os cirurgiões dentistas desenvolvam ações educativas para socializar os saberes } \\
\text { para os futuros profissionais de educação física. } \\
\text { Uso de protetores bucais. }\end{array}$ \\
\hline Francisco et al. (2016) & Devem-se promover campanhas educativas, bem como inclusão da temática no currículo. \\
\hline Jorge et al. (2009) & Uso de protetores bucais. \\
\hline $\begin{array}{l}\text { Mohandas e Chandan } \\
\text { (2009) }\end{array}$ & $\begin{array}{l}\text { Devem ser implementados programas educativos para aprimorar o conhecimento e a } \\
\text { conscientização dos professores. }\end{array}$ \\
\hline $\begin{array}{l}\text { Lempke, Schmidt e } \\
\text { Lynall (2020) }\end{array}$ & $\begin{array}{l}\text { Avaliações multidimensionais devem ser utilizadas na prática para garantir o diagnóstico ideal e } \\
\text { Gestão de situações de trauma. }\end{array}$ \\
\hline Silva et al. (2013) & $\begin{array}{l}\text { Implantação de programas educativos e de formação em primeiros socorros relacionados à } \\
\text { avulsão dentária. }\end{array}$ \\
\hline Santos et al. (2011) & a (paródia) como instrumento para aprendizagem de primeiros socorros. \\
\hline
\end{tabular}

Fonte: Dados de Pesquisa (2020). 
Diante dos achados desta bibliometria, notou-se que os temas versaram entre conhecimentos, competências e estratégias de intervenção, visto que tanto professores de educação física, quanto alunos necessitam de aquisição de saberes gerais no âmbito do atendimento aos primeiros socorros. Independente da área de formação, Nóbrega, Bezerra e Sousa (2015) destacam que identificar e reconhecer previamente o tipo de situação emergencial, bem como poder manejar corretamente é fundamental para minimização de danos. Ademais, importante, ainda, um diálogo multidisciplinar com profissionais da odontologia e de outras áreas.

\section{Conclusão}

O estudo propiciou identificar o perfil das publicações sobre primeiros socorros na educação física. Portanto, constatou-se atualidade no tema (2009-2020) e interesse internacional, pois a base de dados em destaque foi a MEDLINE e os periódicos internacionais, com destaques para o European Journal of Paediatric Dentistry e a Dental Traumatology.

Também, a maior parte das produções era da área de odontologia, redigidos por 41 autores diferentes e por brasileiros, contudo, um autor polonês foi quem mais publicou sobre a temática. Quanto ao Qualis Capes, as revistas variaram entre A1 e B4. Constatou-se, ainda, que a maioria caracterizou-se como estudo transversal e sobre traumas dentários. Os termos mais citados nos títulos e descritores foram conhecimento, educação e educação física e as temáticas versaram, em geral, sobre as estratégias de intervenção.

Assim, pelo pequeno número de publicações sobre o objeto de estudo, sugere-se o desenvolvimento de novas pesquisas, especialmente entre os profissionais da educação física; ainda, pelos demais achados, especialmente referentes ao baixo conhecimento em primeiros socorros, é fundamental adotar estratégias educativas para qualificar os profissionais e os estudantes da área de educação física, bem como estimular o uso de protetores bucais quando for realizar treinamentos esportivos.

\section{Referências}

Antunes, L. A. A., Souza, H. M. R., Gonçalves, P. H. P. Q., Crespo, M. A., \& Antunes, L. S. (2016). Trauma dental e protetor bucal: conhecimento e atitudes em estudantes de graduação em Educação Física. Revista Brasileira de Educação Física e Esporte, 30(2), 287-294. https://www.scielo.br/pdf/rbefe/v30n2/1807-5509-rbefe-30-2-0287.pdf.

Becker, K. E., Molina, F. C., \& Nunes, C. B. (2017). Primeiros socorros nas escolas: opção ou necessidade? Anais do Seminário Internacional de Educação (SIEDUCA), 2(1), 1-5. https://www.ulbracds.com.br/index.php/sieduca/article/view/1272/203.

Brasil (2003). Manual de Primeiros Socorros: Fiocruz

Chueke, G. V., \& Amatucci, M. (2015). O que é bibliometria? Uma introdução ao Fórum. Internext, 10(2), 1-5. https://internext.espm.br/internext/article/view/330/233.

Coelho, J. P. S. L. (2015). Ensino de primeiros socorros nas escolas e sua eficácia. Rev Cient ITPAC, 8(1), 1-4. https://assets.unitpac.com.br/arquivos/Revista/76/Artigo_7.pdf.

Cunha, R. A. D. (2010). A "emergência” das emergências e primeiros socorros na Educação Física escolar: construindo aproximações. Trabalho de Conclusão de Curso (Graduação em Educação Física) - Universidade Federal de Santa Catarina, Florianópolis, Espírito Santo, Brasil. https://repositorio.ufsc.br/bitstream/handle/123456789/191439/Rafael\%20Amaral\%20da\%20Cunha\%20PRONTO.pdf?sequence=1\&isAllowed=y.

Donaldson, A., \& Finch, C. F. (2012). Identifying context-specific competencies required by community Australian Football sports trainers. British Journal of Sports Medicine, 46(10), 759-765. https://bjsm.bmj.com/content/46/10/759.short.

Eiras, F. C. S, Tomomitsu, H. T. A., Linares, I. M. P., \& Carvalho, M. M. (2017). Evolução das pesquisas de gestão de projetos: um estudo bibliométrico do International Journal of Project Management. Revista Gestão da Produção Operações e Sistemas, 12(1), 211-234. https://revista.feb.unesp.br/index.php/gepros/article/view/1617/763.

Emerich, K., Wlodarczyk, P., \& Ziolkowski, A. (2013). Education of Sport University students regarding first-aid procedures after dental trauma. Eur $J$ Paediatr Dent, 14(1), 37-41. http://admin.ejpd.eu/download/EJPD_2013_01_07.pdf.

Francisco, S. S., Amaral, R. C., Vieira, L. M. M., Pereira, C. K., \& Braga, R. D. M. (2016). Conhecimento de estudantes de Educação Física de Juazeiro do Norte-CE sobre o atendimento emergencial ao trauma dental, avulsão e reimplante dental. J. Health Sci. Inst, 34(2), 75-81. https://www.unip.br/presencial/comunicacao/publicacoes/ics/edicoes/2016/02_abr-jun/V34_n2_2016_p75a81.pdf. 
Freitas, R., Neves, R. F. O., \& Gonçalves, V. H. (2018). Utilizando as técnicas de" nuvem de palavras" e clusterização aplicadas as entrevistas dos atletas olímpicos da cidade de São Carlos. Olimpianos-Journal of http://olimpianos.com.br/journal/index.php/Olimpianos/article/view/41.

Jorge, K. O., Ramos-Jorge, M. L., Toledo, F. F., Alves, L. C., Paiva, S. M., \& Zarzar, P. M. (2009). Knowledge of teachers and students in physical education's faculties regarding first-aid measures for tooth avulsion and replantation. Dental Traumatology, 25(5), 494-499. https://pubmed.ncbi.nlm.nih.gov/19754699/.

Lempke, L. B., Schmidt, J. D., \& Lynall, R. C. (2020). Athletic trainers' concussion-assessment and concussion-management practices: an update. Journal of athletic training, 55(1), 17-26. https://pubmed.ncbi.nlm.nih.gov/31855075/.

Losso, E. M., Tavares, M. C. R., Bertoli, F. M. O., \& Baratto-Filho, F. (2011). Traumatismo dentoalveolar na dentição decídua. RSBO Revista Sul-Brasileira de Odontologia, 8(1), e1-e20. http://revodonto.bvsalud.org/pdf/rsbo/v8n1/a19v8n1.pdf

Maldonado, D. T., Silva, S. A. P. S., \& de Jesus Miranda, M. L. (2014). Pesquisas sobre a Educação Física no cotidiano da escola: o estado da arte. Movimento (ESEFID/UFRGS), 20(4), 1373-1395. https://www.seer.ufrgs.br/Movimento/article/view/46072.

Mohandas, U., \& Chandan, G. D. (2009). Knowledge, attitude and practice in emergency management of dental injury among physical education teachers: a survey in Bangalore urban schools. Journal of Indian Society of Pedodontics and Preventive Dentistry, 27(4), 242. https://www.jisppd.com/article.asp?issn=0970-4388;year=2009; volume=27;issue=4;spage=242; epage=248; aulast=Mohandas

Montenegro, E. G., Sousa, H. F., Medeiros, J. O., Santos, L. M. M. A. G., \& Sousa, M. N. A. (2016). Estudos transversais e a prática médica. In: Sousa, M. N. A., \& Santos, E. V. L. (Orgs). Medicina e pesquisa: um elo possível. (pp. 137-152): Editora Prismas.

Moreira, R. F., Figueiredo, R. G., Colodetti, H., \& Miranda, M. S. D. (2015). Traumatismo dentário na adolescência x atividade física. Como orientar e prevenir? Revista Dental Press de Estética, 12(3).

Nóbrega, D. M., Bezerra, A. L. D., \& Sousa, M. N. A. (2015). Conhecimentos, atitudes e práticas em urgência e emergência na atenção primária à saúde. Ciência \& Desenvolvimento-Revista Eletrônica da FAINOR, 8(2), 141-157.

Oleszkiewicz, I., \& Emerich, K. (2015). How to proceed in case of tooth avulsion: State of student knowledge. Eur J Paediatr Dent, 16(2), 103-6. http://admin.ejpd.eu/download/EJPD_2015_2_3.pdf.

Pereira, N. J. G., de Oliveira, J. J. M., Farias, M. F., \& de Araújo, J. M. S. (2020). Perfil das emergências médicas de interesse odontológico atendidas pelo posto de atendimento em primeiros em primeiros socorros-PAPS. Research, Society and Development, 9(8), e391985373. https://doi.org/10.33448/rsdv9i8.5373

Rodrigues, C., \& Viera, A. F. G. (2016). Estudos bibliométricos sobre a produção científica da temática Tecnologias de Informação e Comunicação em bibliotecas. InCID: Revista de Ciência da Informação e Documentação, 7(1), 167-180. https://www.revistas.usp.br/incid/article/download/98761/111654/.

Santos, C. M. P., Oliveira, E. C. S., Sousa, F. N., Tomaz, E. X., Santos, L. C. S., da Silva, J. V. P., \& Sampaio, T. M. V. (2011). A paródia: uma estratégia educativa para conhecimentos relacionados à saúde. Revista Brasileira de Ciência e Movimento, 19(3), 86-98. https://portalrevistas.ucb.br/index.php/RBCM/article/view/2712/2043.

Silva, E. D. D., Siqueira, M. D. F. G., Gomes, M. C., Clementino, M. A., Granville-Garcia, A. F., \& Ferreira, J. M. S. (2013). Conhecimento de alunos do curso de Educação Física sobre avulsão dentária. Arquivos em http://revodonto.bvsalud.org/scielo.php?script=sci_arttext\&pid=S1516-09392013000400004.

Silva, L. G. S., Costa, J. B., Furtado, L. G. S., Tavares, J. B., \& Costa, J. L. D. (2017). Primeiros socorros e prevenção de acidentes no ambiente escolar: intervenção em unidade de ensino. Enferm Foco [Internet], 8(3), 25-29. https://pdfs.semanticscholar.org/a7a8/a9c3df83d61c6a6b3cbdacd89b070ff3339f.pdf.

Sousa, M. N. A., Almeida, F. F. F., Melo, A. C., \& Almeida, E. P. O. (2019). Produção e acesso as redes sociais científicas por doutores docentes de Instituição de Ensino Superior. Revista Brasileira $\quad$ de https://www.gvaa.com.br/revista/index.php/REBES/article/view/6702/5717.

Sousa, M. N. A. (2020). Trilhas acadêmicas: caminhos para a concepção, execução e publicação de artigos científicos: Editora CRV.

Souza, P. D., \& Tibeau, C. (2008). Acidentes e primeiros socorros na Educação Física escolar. Revista Digital-Buenos Aires, 13(127). https://www.efdeportes.com/efd127/acidentes-e-primeiros-socorros-na-educacao-fisica-escolar.htm.

Traebert, J., Marcon, K. B., \& Lacerda, J. T. D. (2010). Prevalência de traumatismo dentário e fatores associados em escolares do município de Palhoça (SC). Ciência \& Saúde Coletiva, 15, 1849-1855. https://www.scielosp.org/article/csc/2010.v15suppl1/1849-1855/pt/.

Vergotine, R. J., \& Govoni, R. (2010). Public school educator's knowledge of initial management of dental trauma. Dental Traumatology, 26(2), 133-136. https://onlinelibrary.wiley.com/doi/abs/10.1111/j.1600-9657.2009.00854.x. 